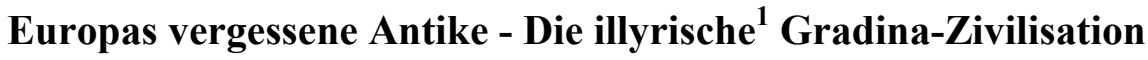

\author{
Anto Pavić
}

Ein in der europäischen Archäologie bislang wenig bekanntes Denkmal ist die Anlage der „gradina“. ${ }^{2}$ Dabei handelt es sich um ein monumentales Bauwerk auf Anhöhen mit einer oder mehreren Ringmauern aus großen Steinblöcken. Derartige Stätten erscheinen bereits sehr früh im Illyricum und können dem bisherigen Forschungsstand nach zu urteilen jeweils in Höhensiedlungen, Burgberge (Akropoleis), Wallburgen (Kastelle) sowie in „öffentliche Denkmäler“ oder Heiligtümer unterschieden werden. Das für den antiken Westbalkan charakteristische Bauwerk soll nun erstmals in seinen Grundzügen gebietsübergreifend vorgestellt werden. Zudem werden Beispiele aus den Bereichen Siedlungsstruktur und Urbanistik entnommen. In Hinblick auf eine über tausendjährige Kulturgeschichte der verschiedenen Landschaften entlang der Adria sowie des Dinarischen Hinterlandes aus vorrömischer Zeit wird zunächst ein zeitlicher Abriss zur historischen Entwicklung der Region gegeben.

\section{Historische Entwicklung}

Der aufgrund seiner klimatischen Verhältnisse sowie reicher Erz- und Edelmetallvorkommen attraktive ostadriatische Raum hat bereits in der griechischen Mythologie Erwähnung gefunden. So gibt es eine Anzahl mythologischer Erzählungen das Adriatische Meer betreffend (Io und die „große Bucht der Rhea“, Kadmos und Harmonia bei den Encheleern und in der Bucht von Rhizon, Jason und die Argonauten entlang der Ostadria an Pola, Mentoriden, Hylloi, Issos, Dyskelados, Pityei, Korkyra melaina, Melita, Salangon vorbei, Medea und die Apsyrtiden, Elektriden, Antenor und die Enetoi, Diomedes und die Adria) sowie von Siedlungsgründungen seitens heroischer Gestalten (Pola, Korkyra melaina, Rhizon, Olcinium, Orikon, Lychnidos). In archaischer Zeit beschränkte sich die griechische Kolonisierung in der Adria jedoch zunächst auf das korinthische Epidamnos / Dyrrhachion (Durres, Albanien), das gemeinsam von Korinth und Korkyra gegründete Apollonia (Fieri, Albanien) sowie das knidische Korkyra melaina (Korčula, Kroatien). Im 4. Jh. v. Chr. entstanden aufgrund der Expansionspolitik Dionysios' I. aus Syrakus neben verschiedenen Gründungen auf der italischen Seite auch griechische Siedlungen auf den kroatischen Inseln im heutigen Mitteldalmatien, so z.B. Issa auf der Insel Vis. Im selben Jahrhundert gründeten Kolonisten aus Paros die Stadt Pharos auf der Insel Hvar. Issa gründete ihrerseits am Ende des 4. Jh. v. Chr. eine weitere Siedlung auf Korkyra melaina (Korčula) sowie im 3. Jh. v. Chr. die Kolonien Tragourion (Trogir) und Epetion (Stobreč) in der Nähe von Split. Darüber hinaus gibt es sowohl archäologische als auch epigraphische Hinweise für einen issäischen

\footnotetext{
${ }^{1}$ In diesem Fall ist ,illyrisch“ als allgemeiner Sammelbegriff für die verschiedenen ostadriatischen oder westbalkanischen Kulturen, Landschaften sowie ethnischen Gruppen gemeint und nicht mit dem Ethnos-Begriff zu verwechseln, welcher im Zusammenhang mit der von PARZINGER 1991 gelösten Illyrer-Frage eindeutig der Gruppe Glasinac-Burrel zugeschrieben wurde.

${ }^{2}$ Im Rahmen meines Dissertationsprojektes über ,Illyricum, Griechenland, Rom - Kontakt induzierter Wandel in den Stadtzentren der Ostadriatischen Antike“" wurden Bosnien und Herzegowina, Kroatien und Mazedonien sowie als Teilnehmer des fachwissenschaftlichen Kurses 2009 des Deutschen Archäologischen Instituts auch Albanien bereist. Die daraus resultierenden Erkenntnisse über das archäologische Denkmal der Gradina-Anlage sowie ihrer weiten Verbreitung auf dem Westbalkan sind als einzigartiges kulturelles Erbe in Europa erkannt worden.
} 
Einfluss in Salona (Solin bei Split) und in Narona (Vid bei Metković). Belegt sind auch griechische Heiligtümer auf dem Palagruža-Archipel (Diomedes insulae) sowie am Kap Ploča bei Rogoznica (Diomedes promunturium).

Durch das griechische Interesse an diesem Raum trat darüber hinaus die ostadriatische Bevölkerung erstmals in die Geschichte ein, obgleich die Namen von Inseln und Völkern aus den mythologischen Erzählungen älteren Ursprungs zu sein scheinen. Wie J. WILKES und T. FISCHER-HANSEN ${ }^{3}$ bereits erkannt haben, trafen die Griechen dabei auf verschiedene protourbane Zivilisationen, welche bis in die Bronzezeit archäologisch zurückzuverfolgen sind (bis etwa 1200 bzw. 1400 v. Chr.). Durch den Kontakt mit der griechischen Kultur soll es nach D. DžINO ${ }^{4}$ in den verschiedenen Landschaften vom 7 . bis 3 . Jh. v. Chr. zu einer territorialen sowie sozialen Transformation gekommen sein. Schließlich führte die über zwei Jahrhunderte andauernde römische Eroberung des westlichen Balkan von 229 v. Chr. bis 9 n. Chr., zunächst auf den Küstenstreifen beschränkt, zu einer Neuordnung der Region. Spätestens unter Caesar soll Illyricum als Provinz eingerichtet worden sein (Bellum Alexandrinum 44-47). Nach weiteren Eroberungen bis zum Donauraum und der Niederschlagung des großen „dalmatisch-pannonischen Aufstands“ 6-9 n. Chr., den Worten Suetons nach dem ,schwersten aller auswärtigen Kriege seit dem Punischen“" (Tib. 16), wurde die Region im 1. Jh. n. Chr. in Dalmatia im Süden und Pannonia im Norden zweigeteilt. Die Halbinsel Istrien wurde der Regio X Venetia et Histria, Südillyrien hingegen der 4. Meris der Provinz Macedonia zugeschlagen. Seitdem gehört der gesamte Westbalkan zum festen Bestandteil des römischen Reiches.

\section{Gradina-Anlagen}

Vom südöstlichen Alpenraum bis hin $\mathrm{zu}$ den antiken Landschaften Makedonien und Epeiros ist unter anderem ein für dieses Gebiet charakteristisches Bauwerk auch der Antike zuzuschreiben. Auf dem Westbalkan allgemein unter dem Begriff „gradina“ bekannt, gehören diese dem Typus der Bauwerke auf Anhöhen mit einer oder mehreren Ringmauern aus großen Steinblöcken an. Derartige archäologische Denkmäler bilden ein regelrechtes Netz in den heutigen Staaten Italien $\left(\right.$ Triester Hinterland ${ }^{5}$ ), Slowenien (westlich und südlich der Sava ${ }^{6}$ ), Kroatien (gesamte Küstenregion und Mittelkroatien ${ }^{7}$ ), Bosnien und Herzegowina (südlich der SavaEbene $^{8}$ ), Serbien (südlich der Donau und westlich der Morava ${ }^{9}$ ), Montenegro ${ }^{10}$, Albanien $^{11}$ (nördlich der Akrokeraunischen Berge und des Vjosë) und Mazedonien ${ }^{12}$ (um den sowie nördlich des Ohrid-See). Die oben beschriebene geographische Verbreitung derartiger Gradina-Anlagen deckt sich darüber hinaus mit jenem Großraum, welcher sowohl in den literarischen Quellen als auch in der modernen Wissenschaft allgemein als „Illyricum“ bekannt ist.

\footnotetext{
${ }^{3}$ WILKES - FISCHER-HANSEN 2004, 321-22.

${ }^{4}$ DŽINO 2005, 48.

${ }^{5}$ MARCHESETTI 1903.

${ }^{6}$ BENAC 1987, 29-177; DULAR - TECCO HVALA 2007.

${ }^{7}$ BATOVIĆ 1973, 5-153; DRECHSLER-BIŽIĆ 1974, 71-79; BENAC 1974, 81-89; BENAC 1987, 317-320; 351-355; 416-424; 459-469; CHAPMAN 1996, 110-157.

${ }^{8}$ MARIĆ 1974, 103-111; ČOVIĆ 1974a, 93-101; BENAC 1987, 506-510; 523-525.

${ }^{9}$ GARAŠANIN 1975, 113-120.

${ }^{10}$ GARAŠANIN 1966, 27-28; MIJOVIĆ-KOVAČEVIĆ 1975.

${ }^{11}$ KORKUTI 1975, 131-136; CEKA 1988, 215-229.

12 GARAŠANIN 1975, 113-120.
} 
Anders als in den übrigen Teilen der antiken Welt, handelt es sich auf dem Westbalkan um strategisch angeordnete Anlagen auf planierten Hügeln, Bergrücken, Inseln sowie Halbinseln, die bevorzugt in fruchtbaren Regionen angelegt wurden. Die Umfassungsmauern bestimmen den je nach Geländeverlauf unterschiedlichen Grundriss. Vornehmlich liegt ihnen ein kreisrunder oder ovaler Plan zugrunde (Abb. 1.). Vereinzelt kommen aber auch geometrische Formen in den Grundrissen vor (z.B. Hochebene Glasinac). Die bislang erhaltenen Mauerzüge besitzen in der Regel eine bemerkenswerte Stärke zwischen drei und sechs Metern, in Ausnahmefällen konnte eine Breite von zehn, fünfzehn und dreißig Metern erreicht werden. ${ }^{13}$ Die Mauern umschlossen jeweils ein Areal von mehreren hundert Quadratmetern bis einigen Quadratkilometern und wurden nach B. KUNTIĆ-MAKVIĆ U.A. ${ }^{14}$ in der einheimischen Bautechnik des Trockenmauerwerks in zwei oder mehreren Schalen errichtet. Die Stirnseiten weisen die jeweils vorherrschende Technik auf. Als charakteristische Merkmale für ,illyrische“ Mauern in klassischer sowie hellenistischer Zeit sind neben den zwei bis drei Meter messenden Seitenlängen sowie der trapezoiden Form der Blöcke, die grob bearbeiteten Frontseiten der Steine sowie die Vermischung zwischen pseudoisodomer und polygonaler Bauweise mit Einklinkungen zu nennen (Abb. 2.). Aufgrund der bemerkenswert hohen Anzahl an „gradina“ genannten Orten ist ein genaues Bild des gesamten Ausmaßes der Verbreitung dieses Bautyps im ostadriatischen Raum bislang nicht bekannt. Während vereinzelte oder auch mehrere Gradinen lokalisiert und in wissenschaftlichen Arbeiten behandelt wurden, haben in einzelnen Regionen regelrechte Zählungen stattgefunden. Allen Schätzungen zufolge ist im gesamten Raum vom Golf von Triest bis zum Neretva-Tal und dem mittleren Lauf der Drina, also lediglich im nördlichen Teil des Illyricum, von insgesamt über 2500 derartigen Anlagen auszugehen. ${ }^{15}$ Zählungen aus den weiter südlich gelegenen Gebieten sind, soweit hier bekannt, bislang nicht erfolgt. Derartige Anlagen werden aufgrund des Fundmaterials in das erste (alle ,illyrischen“ Kulturen ${ }^{16}$ ), zweite (z. B. kašteljerska kultura / cultura dei castellieri in Istrien ${ }^{17}$ ) sowie das Ende des dritten ( $\mathrm{z}$. B. Cetina-Kultur ${ }^{18}$ ) vorchristlichen Jahrtausends datiert.

Dem bisherigen Forschungsstand nach zu urteilen, können diese Stätten nach der strategischen Anordnung der Anlagen, der Art der Befestigungen sowie der planmäßigen Gestaltung ihrer Innenbebauung jeweils in Höhensiedlungen, Burgberge (Akropoleis), Wallburgen (Kastelle) sowie in „öffentliche Denkmäler“ oder Heiligtümer unterschieden werden. Die Höhensiedlungen lassen sich des Weiteren in Zentral- und Satellitensiedlungen gliedern.

\footnotetext{
${ }^{13}$ MIHOVILIĆ 2005, 31.

${ }^{14}$ KUNTIĆ MAKVIĆ 2007, 22.

${ }^{15}$ Demnach wurden von MARCHESETTI 1903 für die Halbinsel Istrien und dem Triester Hinterland zusammen 400 castellieri oder Gradinen angegeben. In dem der Eisenzeit gewidmeten Band der Praistorija Jugoslavenskih Zemalja - Vorgeschichte der Jugoslawischen Länder (vgl. GABROVEC ČOVIĆ 1987, 912) werden für Liburnien 600, für das japodische Kernland 200, für das heutige Bosnien und Herzegowina um die 1000, für das slowenische Verbreitungsgebiet um die 100, für das Gebiet der Daorser 60, für die Region Šibenik 30 und für die Region Knin 35 Gradinen erwähnt. Demgegenüber sind bislang lediglich in Istrien 350, in der Lika 97 (vgl. DRECHSLER-BIŽIĆ 71-79), in Norddalmatien 35 (vgl. BATOVIĆ 1968, T. 1), im Archipel von Zadar 46 (vgl. BATOVIĆ 1973, Karta 5) in Südwestbosnien 108 (vgl. WILKES 1992, ), in Mittelbosnien 120 (vgl. DOMIĆ KUNIĆ 82), in der Hochebene von Glasinac 47 (vgl. ČOVIĆ 1974a, 93, 100-101) und im Neretva-Tal 57 (vgl. MARIĆ 1974, 103-110, Karta 1) Anlagen tatsächlich dokumentiert worden.

${ }^{16}$ Vgl. BENAC 1987, 289-530, 575-650.

${ }^{17}$ GABROVEC 1983, 46-49; MIHOVILIĆ 2005, 31-37.

${ }^{18}$ MAROVIĆ - ČOVIĆ 1983, 191-231; GOVEDARICA 2006, 27-41.
} 
$\mathrm{Zu}$ den Höhensiedlungen zählen großflächige Anlagen, von welchen einige Exemplare jeweils eine Siedlungskontinuität bis in die römische Zeit oder sogar bis heute aufweisen (z. B. Parentium / Poreč, Ruginium / Rovinj, Pola / Pula, Nesactium, Albona / Labin, Aenona / Nin, Iader(a) / Zadar, Asseria, Varvaria / Bribir, Tragourion / Tragurium / Trogir, Epetion / Stobreč, Škrip, Pharos / Pharia / Starigrad). Das sind Stadtanlagen, um welche sich während der Eisenzeit regelrechte Gradina-Ketten gebildet haben. Diese schützten nach neueren Erkenntnissen das Territorium ihres Gemeinwesens und waren der jeweiligen führenden Gradina als wirtschaftlichem, politischem sowie kulturellem Zentrum der gesamten Gemeinde untergeordnet. ${ }^{19}$ Bereits während der vorangehenden Bronzezeit haben sich in bestimmten Regionen der Ostadria viele kleine Gemeinwesen mit einer als Hierarchie interpretierbaren inneren Gliederung in zentrale Siedlungen und ihnen untergeordnete Gemeinden gebildet (z.B. in Istrien, ${ }^{20}$ Glasinac ${ }^{21}$ ).

Es kommen ebenso Siedlungen vor, welche einen Burgberg in der Mitte oder am Rande ihrer in verschiedene Ebenen oder Terrassen eingeteilten Areale umschließen und demnach, neben einem Burgberg, in eine Oberstadt und mancherorts auch in eine Unterstadt aufgeteilt werden können (z. B. Monkodonja, Arupium, Pod, Delmion, Daorson, Meteon, Lissos). Während sich im Bezug auf die letztgenannten Siedlungen offensichtlich Analogien zu Städten mit einer ähnlichen Aufteilung und einer Akropolis als Bekrönung im ägäischen Raum finden, lässt sich im ostadriatischen Raum aufgrund der spezifischen topographischen Situation sowie der im Laufe der Zeit entstehenden differenzierten Gesellschaftsstruktur eine autochthone Entwicklung verfolgen. ${ }^{22}$

Des Weiteren erfüllten einige Gradinen aufgrund der kleineren Dimensionen sowie ihrer strategischen Anordnung um ein bestimmtes Territorium oder entlang wichtiger Handelsrouten die Funktion einer zu Verteidigungszwecken angelegten Wallburg (z.B. Gradinen am Rande der Hochebenen von Gacka, Lika, Livno, Duvno, Glamoč, Kupres, Glasinac sowie entlang vorrömischer Straßenführungen in Histrien oder Liburnien).

Darüber hinaus kann eine sehr große Anzahl an kleineren Gradinen im gebirgigen Landesinneren wohl als „öffentliche Denkmäler“ oder als Heiligtümer (z.B. Mandina gradina in der Hocheben von Duvno, gradina Vrsnik in der Ostherzegowina) ihrer jeweiligen Gemeinwesen angesehen werden. Diese Vielzahl an kleineren Anlagen wurde jedoch bislang aufgrund ihrer Dimensionen, ihrer Lage an meist unzugänglichen Orten sowie aufgrund der dort entdeckten tierischen und anderen Abfälle als Refugien in Kriegszeiten oder als Viehgehege gedeutet. ${ }^{23}$ Allerdings werden nach neueren Forschungen von V. GAFFNEY U. A. ${ }^{24}$ die meisten Anlagen besser als ,public monuments“ interpretiert, welche verbunden mit der Kontrolle über ein bestimmtes Territorium durch alltägliche Rituale, die Macht der lokalen Potentaten reflektieren sollten. Dieser Deutung ist wohl eher zuzustimmen, zumal sich die genannten Anlagen einerseits alle an exponierter Stelle befinden und damit weit sichtbar für ein bestimmtes Areal waren, stellte gerade die schwere

\footnotetext{
${ }^{19}$ KUNTIĆ MAKVIĆ 2007, 22.

${ }^{20}$ HÄNSEL 2005, 13.

${ }^{21}$ TRUHELKA 1893, 65.

${ }^{22}$ Es sei an dieser Stelle bemerkt, dass der Typus der Gradina im Grunde mit den griechischen Akropoleis vergleichbar ist, zumal letztgenannte ursprünglich ebenso als (mykenische) Höhensiedlungen dienten.

${ }^{23}$ Vgl. DRECHSLER-BIŽIĆ 1974, 73; SUIĆ 1975, 9-36; BENAC 1987, 459-469; SUIĆ 2003, 63-81.

${ }^{24}$ Vgl. GAFFNEY 2001, 152; GAFFNEY 2002, 25-43.
} 
Zugänglichkeit die herausragende Lage sicher. Andererseits sprechen die kleineren Dimensionen des jeweiligen oberen Plateaus derartiger Gradinen sicherlich gegen ihre Funktion als Siedlung. Auch ihre Interpretation als Refugien ist in Anbetracht einer ohnehin großen Dichte an befestigten Höhensiedlungen wenig überzeugend. Ebenso wenig sprechen die meterdicken Mauern und die meist unzugängliche Lage kaum dafür, dass die Gradinen als Tiergehege genutzt wurden. Vielmehr liefern die gefundenen tierischen Abfälle zusammen mit der Lage auf Anhöhen in Analogie zu den frühen Heiligtümern im ägäischen Raum Argumente für eine sakrale Nutzung der in kleineren Dimensionen angelegten Gradinen. Zudem bekommen die jeweiligen Orte durch den architektonischen Rahmen eine besondere Bedeutung zuerkannt. Schließlich wurden auch die vormaligen auf Anhöhen gelegenen helladischen Siedlungen und Heiligtümer seit der archaischen Zeit als griechische Akropoleis mit einem besonderen Bauwerk als Denkmal versehen. ${ }^{25}$

\section{Siedlungsstruktur}

Obgleich im Illyricum die geographischen Besonderheiten in ähnlicher Weise wie in Griechenland das Entstehen kleinräumiger politischer Einheiten, in fruchtbaren Ebenen und von Gebirgen umschlossen oder auf Inseln gelegen, förderten, lassen die auf Gradinen basierenden ostadriatischen Gemeinwesen eine komplex gegliederte Siedlungsstruktur erkennen. Ein Gradina-Gemeinwesen bestand demnach aus einem flächenmäßig großen Zentralort an exponierter Stelle sowie mehreren kleineren strategisch günstig angelegten Siedlungen. Darüber hinaus wurde das gesamte Gemeinwesen durch Wallburgen beschützt. Aus Verteidigungsgründen sowie zu Kommunikationszwecken hatten Zentralort, Satellitensiedlungen und Wallburgen Sichtkontakt zueinander (Abb. 3.). Im Gegensatz zur Polis ist ein GradinaGemeinwesen demnach in Analogie zu jenen später im südlichen Illyricum historisch belegten Siedlungen annähernd mit einem kouvov (z.B. Byllis, Amantia) oder einer civitas (z.B. liburnische civitates) zu vergleichen. ${ }^{26}$

Als Träger der auf Gradina-Anlagen basierenden Zivilisation sind verschiedene autochthone Kulturen aus vorrömischer Zeit auszumachen, welche aus antiken Quellen jeweils als ethnische sowie politische Gruppen namentlich bekannt sind. Entlang der Ostküste des Adriatischen Meeres sowie im Dinarischen Hinterland können nach dem jetzigen Stand der Forschung Kulturen bis in die späte Bronzebzw. frühe Eisenzeit zurückverfolgt werden. Nach Ausweis der Funde sind zu dieser Zeit bereits Histrier ${ }^{27}$, Liburner ${ }^{28}$, Japoden ${ }^{29}$, „Delmater“ (Mitteldalmatinische Gruppe $^{30}$ ), „Ostdalmater“ (Mittelbosnische Gruppe ${ }^{31}$, später auch Daesitiaten, Maezaeer, Ditionen) und Illyrer (Kulturgruppe Glasinac-Mati ${ }^{32}$ bzw. GlasinacBurrel $^{33}$ ) archäologisch fassbar sowie einige ihrer Volksnamen seit dem 6. Jh. v. Chr. literarisch belegt (Hekataios, Fr.8). ${ }^{34}$ Demnach können bereits spätbronzezeitliche Gradina-Siedlungen in einem bestimmten Territorium jeweils diesen antiken Völkern zugeordnet werden (Abb. 4.).

\footnotetext{
${ }^{25}$ Vgl. KOLB 2005, 70-72.

${ }^{26}$ DŽINO 2005, 49.

${ }^{27}$ Vgl. GABROVEC - MIHOVILIĆ 1987, 293-338; STARAC 1999, 11-15.

${ }^{28}$ Vgl. BATOVIĆ 1987, 339-390; STARAC 2000, 7-22.

${ }^{29}$ Vgl. DRECHSLER-BIŽIĆ 1987, 391-441; OLUJIĆ 2007; BALEN-LETUNIĆ 2004, 211-257.

${ }^{30}$ Vgl. ČOVIĆ $1987 \mathrm{a}, 442-480$.

${ }^{31}$ Vgl. ČOVIĆ $1987 b, 481-530$.

${ }^{32}$ Vgl. ANDREA 1985, ; ČOVIĆ 1987c, 575-643.

${ }^{33}$ Vgl. PARZINGER 1991, 205-261.

${ }^{34}$ Vgl. ŠAŠEL KOS 2005, 115-132; KUNTIĆ MAKVIĆ 2007, 21.
} 
Die führenden Gradina-Siedlungen in Histrien, Liburnien, in Delmatien und in Illyrien beherrschten genauso wie die zeitgenössischen etruskischen Städte und wie die griechischen Stadtstaaten jeweils ein umliegendes Territorium. Die Küstenstädte von der Halbinsel Istrien im Norden bis zum Epeiros im Süden lagen in der Regel genauso wie diejenigen in Etrurien aus Sicherheitsgründen immer einige Kilometer im Landesinneren (z.B. Picugi, Nesactium, Asseria, Varvaria, Ošanići, Meteon, Zgërdhesh, Byllis, Amantia), während sie ihre eigenen Häfen und Handelsniederlassungen (z.B. Parentium, Budava, Pakoštane, Tragourion, Salona) unmittelbar am Meer besaßen. ${ }^{35}$ Im Falle der Adriatischen Ostküste beschützten und kontrollierten zudem zahlreiche kleinere Festungsanlagen den Schiffsverkehr in diesen Gewässern (z.B. auf den Inseln Ugljan, Iž, Pašman, Školj bei Pakoštane). Im Landesinneren hingegen haben sich während dieser Zeit in Japodien, Delmatien sowie in Ostdalmatien (Maezaeer, Ditionen, Daesitiaten) regelrechte „Wallburgketten gebildet, die das Territorium ihrer Gemeinden schützten und einer führenden“ Gradina als wirtschaftlichem, politischem und religiös-kulturellem Zentrum untergeordnet waren (z. B. Arupium und Gacko Polje, Delmion und Duvanjsko polje). ${ }^{36}$

\section{Urbanistik}

Nach dem bisherigen Forschungsstand ist nur sehr wenig über den Bautypus der Gradina bekannt, welcher seit der späten Bronze- bzw. frühen Eisenzeit den verschiedenen ,illyrischen“ oder ostadriatischen Völkern zugeschrieben werden kann. Nach Ausweis der bislang aus dieser Frühzeit stammenden Funde gibt es keine großen Bevölkerungskonzentrationen unter den „Illyrern“, die sich etwa mit denen der später erscheinenden oppida bei den keltischen Völkern vergleichen lassen. ${ }^{37}$ Dies ist zum einen sicherlich auf die geographischen Gegebenheiten dieser Region, zum anderen auf die komplex gegliederten Siedlungsstrukturen zurückzuführen.

Darüber hinaus ist sehr wenig über die Infrastruktur ihrer Siedlungen bekannt, welche sich im Laufe der Jahrhunderte in verschiedenen Phasen der Urbanisation entwickelten. Während im Norden des Illyricum bereits seit der frühen Bronzezeit planmäßig angelegte Siedlungen ein stadtähnliches Bild erkennen lassen (z.B. Monkodonja), entwickelten sich die Anlagen im Süden ab dem 5. Jh. v. Chr. nach griechischem Vorbild zu Städten (z.B. Amantia, Byllis, Lissos, Scodra, Meteon, Rhizon, Ošanići).

Dem archäologischen Befund nach $\mathrm{zu}$ urteilen, lassen sich in den oberadriatischen Landschaften Histrien $\left(\right.$ Parentium $^{38}$, Nesactium ${ }^{39}$ ) und Liburnien (Aenona $^{40}$, Iader(a) ${ }^{41}$, Asseria ${ }^{42}$, Varvaria ${ }^{43}$, Radovin ${ }^{44}$ ) Stadtanlagen mit einem zentralen offenen Platz ausmachen. Lediglich in Asseria und Varvaria befindet sich das städtische Zentrum jeweils am südlichen Rand des Plateaus der Höhensiedlungen.

\footnotetext{
${ }^{35}$ Vgl. ZANINOVIĆ 2005, 121-123.

${ }^{36}$ Vgl. KUNTIĆ MAKVIĆ 2007, 22.

${ }^{37}$ WILKES 1992, 226.

${ }^{38}$ Vgl. BALDINI 1994, 125-136; BALDINI 1999-2000, 451-457.

${ }^{39}$ Vgl. MATIJAŠIĆ 1990, 635-652; MATIJAŠIĆ 1998, 9; LETZNER 2005, 89-101.

${ }^{40}$ Vgl. WILKES 1969, 205; BATOVIĆ 1970, 33-48; WILKES 1992, 56; ILAKOVAC 1998, 1-14; BELOŠEVIĆ 2000, 111-121; KOLEGA 2001, 83-95; KOLEGA 2005, 90-97.

${ }^{41}$ Vgl. ILAKOVAC 1962, ; SUIĆ 2003, 245-246, 251 ; WILKES 1969, 207, 368.

${ }^{42}$ LIEBL - WILBERG 1908, 17; WILKES 1969, 369; SUIĆ 2003, 249.

${ }^{43}$ BATOVIĆ 1968, 85-92; SUIĆ 1968, 217-234; MARIN 1968/69, 155-176; BATOVIĆ 1980, 55-94; KOROŠEC 1980, 95-164.

${ }^{44}$ BATOVIĆ 1968, 73-74; WILKES 1969, 56.
} 
In den histrischen Städten Parentium und Nesactium sowie in der liburnischen Stadt Aenona lässt sich jeweils am Rand der Platzanlage zudem eine Kultstätte ausmachen. Die in römischer Zeit jeweils mit Tempeln versehenen Stellen weisen darüber hinaus archäologische Reste von Vorgängerbauten auf. Demzufolge erscheinen auf dem Gebiet des antiken Illyricum im Zeitraum vom 12.-9. Jh. v. Chr. zunächst neue, planmäßig angelegte Städte. Im Unterschied $\mathrm{zu}$ den vorangehenden befestigten Siedlungen besitzen die Anlagen in der Küstenregion des Adriatischen Meeres nun eine besonders ausgesparte Fläche für gesellschaftliche Aktivitäten. ${ }^{45}$

Wie bereits J. WILKES ${ }^{46}$ erkannte, lassen sich dem archäologischen Befund sowie der topographischen Situation zufolge um die oberadriatischen städtischen Plätze konzentrisch angelegte Häuserreihen mit radial vom Zentrum aus zur Stadtmauer hin führenden Straßen rekonstruieren (,spider's web“). Die histrischen sowie liburnischen Städte bilden damit sehr früh eine für ihre Landschaften charakteristische Infrastruktur, welche im antiken Mittelmeergebiet ihresgleichen sucht (Abb. 5.).

Im Hinterland der mittleren Adria, im antiken Ostdalmatien (Mittelbosnien), hingegen findet sich eine im 12. Jh. v. Chr. planmäßig angelegte urbane Siedlung, welche bereits in der archaischen Zeit eine bedeutende Umwandlung erfahren hat. In diesem Fall handelt es sich um ein Stadtgebiet, welches durch zwei sich im rechten Winkel kreuzende Hauptstraßen in vier Teile gegliedert wurde. Die einzelnen Viertel wurden ihrerseits durch ein orthogonales Straßensystem unterteilt. Am Schnittpunkt der beiden Hauptstraßen lässt sich ein rechtwinklig angelegter Platz in zwei Phasen gliedern. Obgleich von den Dimensionen her eher klein, gilt die ostdalmatische (mittelbosnische) Siedlung im heutigen Pod bei Bugojno ${ }^{47}$ (Abb. 6.) als bislang frühester Beleg für eine befestigte Stadt mit einem orthogonalen Rastersystem sowie für eine planmäßige Errichtung eines rechtwinklig angelegten urbanen Zentrums im antiken Europa.

In dem an der unteren Ostadria gelegenen Südillyrien hingegen erscheinen ab der Mitte des 5. Jhs. v. Chr. neue planmäßig angelegte Städte. Diese entstanden entweder in bereits bestehenden protourbanen Siedlungen oder sind als Neugründungen konzipiert worden. Die in der Gradina-Tradition weiterhin auf Anhöhen gelegenen Siedlungen (z.B. Byllis, Nikaia) wurden jeweils mit einem Theater, Sitzreihen eines Stadions, Säulenhallen sowie mit gerade verlaufenden Stadtmauern und Türmen versehen (Abb. 7.).

In Bezug auf die Wohnarchitektur ist ein ähnliches Bild zu konstatieren. Stellten nach N. CEKA ${ }^{48}$ die Kolonien Dyrrhachion und Apollonia einen weiteren urbanisierenden Faktor dar, hat eine Urbanisierung nach griechischem Vorbild von Süden nach Norden stattgefunden. Während in Liburnien oder in Japodien bislang eine dichte Besiedlung mit einfachen Hausgrundrissen aus einem oder mehreren Räumen $\mathrm{zu}$ verzeichnen ist, erscheinen in Illyrien dagegen größer angelegte Wohnbauten. So wurden auf der Gradina Ošanići („Daorson“) in der Ostherzegowina nördlich und nordöstlich der sogenannten Akropolis bislang fünfzehn großzügig angelegte Terrassen mit weiten Räumen und offenen Höfen freigelegt. ${ }^{49}$ Weiter

\footnotetext{
${ }^{45}$ Es handelt sich hierbei um die für protourbane Siedlungen im Illyricum herausgearbeiteten Ergebnisse meiner noch nicht publizierten Dissertation.

${ }^{46}$ WILKES 1992, 56, 227.

${ }^{47}$ ČOVIĆ 1974b, 121-129.

${ }^{48}$ CEKA 1988, 215-217.

${ }^{49}$ MARIĆ 1995, 40.
} 
südlich im heutigen Albanien lässt der archäologische Befund nach N. CEKA ${ }^{50}$ keine einem Städtebauplan untergeordnete Typisierung der Wohnhäuser vermuten. Die Übernahme der Peristyl-Häuser geht mit dem Versuch einher, die lokalen Typen durch eine bestimmte Anordnung um den Herdraum zu verbessern. So finden sich Beispiele einer linearen Aufstellung an der Seite einer Halle (Selca, Margellic, Mashkjeze), der Anordnung an beiden Seiten eines Korridors (Antigonea, Klos, Borsh) oder der Errichtung übereinander auf einem schrägen Gelände (Aitoi, Mashkjeze).

Neben den Völkern in Südillyrien scheinen die Liburner die urbanisierteste Siedlungsstruktur im vorrömischen Illyricum besessen zu haben. In römischer Zeit ist keine einzige Stadt auf ihrem Territorium gegründet worden. Vielmehr erwarben 37 Zentralorte liburnischer Gradina-Gemeinwesen den Status einer Kolonie oder den eines Munizipium und wurden nach dem römischen Modell umgeformt (z. B. Aenona, Iader(a), Asseria, Vavaria) (Abb. 8.).

\section{Schlussbetrachtung}

Monumentale Anlagen auf planierten Bergrücken von mehreren hundert Metern Höhe zu bauen und mit drei bis dreißig Meter starken Mauern aus großen Steinblöcken zu befestigen, muss als zivilisatorische Leistung anerkannt werden. Die zentrale Gewalt, die für die Errichtung derart großer Bauprojekte vonnöten war, und die erkennbare komplex gegliederte Siedlungsstruktur sind Teil des sich zu Beginn der frühen Bronzezeit konsolidierenden Gemeinwesen, welche als GradinaZivilisation zu bezeichnen sind. Als Träger der auf Gradina-Anlagen basierenden Zivilisation sind zumindest ab der mittleren bzw. späten Bronzezeit verschiedene Kulturgruppen $\mathrm{zu}$ erkennen, welche kontinuierlich bis in die römische Kaiserzeit verfolgt werden können und in der jeweiligen Landschaft seit der archaischen Zeit auch namentlich bekannt sind.

Nach den obigen Ausführungen sind die wohl in der Bronzezeit entstandenen Gradina-Gemeinwesen (,Koıvov“/ „,civitas“) in den jeweiligen später erscheinenden Ethnos-Namen der Histrier, Liburner, Japoden, Delmater, Illyrer, Taulanter u.a. als politische und / oder ethnische weit ausgedehnte Territorial-Gemeinwesen anzusehen. Dabei bildete die Einheit des Gradina-Gemeinwesens das Fundament eines jeden neu entstandenen Königreichs oder Bündnisses, welches wiederum nach dem Zerfall einer derartigen Staatenbildung anhand der Aufteilung eines bestimmten Gebiets in der Vielzahl der lokalen Zentren ersichtlich ist.

Darüber hinaus gibt die Infrastruktur auf den bislang wenigen aufgedeckten Gradinen bereits sehr früh Einblicke in eine höher entwickelte Organisationsform, wie sie in der antiken Welt des übrigen Europa bislang unbekannt geblieben ist (histrische und liburnische Städte) oder erst wesentlich später erscheint (ostdalmatische Stadt bei Pod).

Neue Ausgrabungen mögen unser Bild des antiken Westbalkan ändern. Beim momentanen Erkenntnisstand ist festzuhalten, dass die illyrische Gradina-Zivilisation bestimmt wird von vielen, komplex gegliederten Gemeinwesen mit hoher Organisationsform in einem weitläufigen, von Gebirgen durchzogenen Land.

\footnotetext{
${ }^{50}$ CEKA 1988, 226.
} 


\section{Verzeichnis der abgekürzt zitierten Literatur}

ANDREA 1985

Z. Andrea, Mbi gjenezën dhe vijmësinë e kulturës së Matit në epokën e bronzit. A propos de la genèse et de la continuité de la culture de Mat à l'époque du bronze, in: Iliria 15,2, 1985, 170-172.

\section{BALDINI 1994}

M. Baldini, Marafor, forum Coloniae Iuliae Parentium - Marafor, Stadtforum Coloniae Iuliae Parentium, in: Arheološka istraživanja u Istri. Znanstveni skup, Poreč 22. - 26. rujna 1994 (Zagreb 1997) 125-136.

\section{BALDINI 1999-2000}

M. Baldini, Parentium - prilozi čitanju urbane kronologije, in: Opus. archaeol. 23-24, 1999-2000, 451-457.

\section{BALEN-LETUNIĆ 2004}

D. Balen-Letunić, Japodi, The Iapodes, Die Japoden, in: A. Rendić-Miočević (Hrsg.), Ratnici na razmeđu istoka i zapada (Zagreb 2004), 211-257.

\section{BATOVIĆ 1968}

Š. Batović, Istraživanje ilirskog naselja u Radovinu, in: Diadora 4, 1968, 5374.

\section{BATOVIĆ 1970}

Š. Batović, Istraživanje liburnskog naselja u Ninu 1969. godine -

Ausgrabungen der liburnischen Siedlung in Nin 1969, in: Diadora 5, 1970, 3348.

\section{BATOVIĆ 1973}

Š. Batović, Prapovijesni ostaci na zadarskom otočju - Les vestiges préhistoriques sur 1'archipel de Zadar, in: Diadora 6, 1973, 5-153.

\section{BATOVIĆ 1980}

S̆. Batović, Istraživanje prapovijesti u Bribiru - Les recherches de la préhistoire a Bribir, in : Diadora 9, 1980, 55-94.

\section{BATOVIĆ 1987}

Š. Batović, Liburnska grupa, in: A. Benac - S. Gabrovec (Hrsg.), Praistorija Jugoslavenskih Zemalja 5, Željezno doba (Sarajevo 1987) 339-390.

\section{BELOŠEVIĆ 2000}

J. Belošević, Arheoloska istrazivanja okolisa crkve sv. Kriza u Ninu, in:

Obavijesti 32, 2000, Nr. 3, 111-121.

\section{BENAC 1974}

A. Benac (Hrsg.), Utvrđena ilirska naselja. Međunarodni kolokvij, Mostar 2426 oktobar 1974 - Agglomérations fortifiées illyriennes. Colloque international, Mostar 24-26 octobre 1974).

\section{BENAC 1983}

A. Benac (Hrsg.), Praistorija Jugoslavenskih Zemalja 4, Bronzano doba (Sarajevo 1983).

BENAC 1987

A. Benac - S. Gabrovec (Hrsg.), Praistorija jugoslavenskih zemalja 5, Željezno doba (Sarajevo 1987).

\section{CEKA 1988}

N. Ceka, Städtebau in der vorrömischen Periode in Südillyrien, in: Akten des XIII. internationalen Kongresses für Klassische Archäologie (Berlin 1988) 215-229. 


\section{CHAPMAN 1996}

J. Chapman-R. Shiel-ŠS. Batović, The changing face of Dalmatia (London 1996) $110-157$.

\section{ČOVIĆ 1974a}

B. Čović, Die Befestigungen und befestigte Siedlungen des Glasinacer Gebiets, in: A. Benac (Hrsg.), Utvrđena ilirska naselja. Međunarodni kolokvij, Mostar 24-26 oktobar 1974 - Agglomérations fortifiées illyriennes. Colloque international, Mostar 24-26 octobre 1974) 93-102.

\section{ČOVIĆ 1974b}

B. Čović, Pod bei Bugojno. Eine befestigte Siedlung der Bronze- und Eisenzeit in Zentralbosnien, in: A. Benac (Hrsg.), Utvrđena ilirska naselja. Međunarodni kolokvij, Mostar 24-26 oktobar 1974 - Agglomérations fortifiées illyriennes. Colloque international, Mostar 24-26 octobre 1974) 121-129.

\section{ČOVIĆ 1983a}

B. Čović, Srednje brončano doba u Istri, in: A. Benac (Hrsg.), Praistorija Jugoslavenskih Zemalja 4, Bronzano doba (Sarajevo 1983) 46-49.

\section{ČOVIĆ 1987a}

B. Čović, Srednjodalmatinska grupa, in: A. Benac - S. Gabrovec (Hrsg.), Praistorija jugoslavenskih zemalja 5 (Sarajevo 1987) 442-480.

\section{ČOVIĆ 1987b}

B. Čović, Srednjobosanska grupa, in: A. Benac - S. Gabrovec (Hrsg..),

\section{ČOVIĆ 1987c}

Praistorija Jugoslavenskih Zemalja 5 (Sarajevo 1987) 481-530.

B. Čović, Glasinačka kultura, in: A. Benac - S. Gabrovec (Hrsg..), Praistorija Jugoslavenskih Zemalja 5 (Sarajevo 1987) 575-643.

\section{DOMIĆ KUNIĆ 2006}

A. Domić Kunić, Bellum Pannonicum (12.-11. pr.Kr.). Posljedna faza osvajanja Južne Panonije, in: VAMZ 39,3, 2006, 59-164.

\section{DRECHSLER-BIŽIĆ 1974}

R. Drechsler-Bižić, Caractéristique des agglomerations fortifiées dans la région centrale des Japodes, in: A. Benac (Hrsg.), Utvrđena ilirska naselja. Međunarodni kolokvij, Mostar 24-26 oktobar 1974 (Agglomérations fortifiées illyriennes. Colloque international, Mostar 24-26 octobre 1974) 71-80.

\section{DRECHSLER-BIŽIĆ 1987}

R. Drechsler-Bižić, Japodska grupa, in: A. Benac - S. Gabrovec (Hrsg.),

Praistorija Jugoslavenskih Zemalja 5 (Sarajevo 1987) 391-441.

\section{DULAR - TECCO HVALA 2007}

J. Dular - S. Tecco Hvala, Southeastern Slovenia in the early iron age.

\section{DŽINO 2005}

Settlement - Economy - Society (Ljubljana 2007).

D. Džino, Illyrian Policy of Rome in the Late Republic and Early Principate (Adelaide 2005).

\section{GABROVEC 1983}

S. Gabrovec, Srednje brončano doba, in: S. Gabrovec (Hrsg.), Praistorija Jugoslavenskih Zemalja 4, Bronzano doba (Sarajevo 1983) 40-51.

\section{GABROVEC - ČOVIĆ 1987}

S. Gabrovec - B. Čović, Zključna razmatranja, in: S. Gabrovec (Hrsg.), Praistorija Jugoslavenskih Zemalja 5, Željezno doba (Sarajevo 1987) 901-928. 


\section{GABROVEC - MIHOVILIĆ 1987}

S. Gabrovec - K. Mihovilić, Istarska grupa, in: S. Gabrovec (Hrsg.), Praistorija Jugoslavenskih Zemalja 5, Željezno doba (Sarajevo 1987) 293-338.

\section{GAFFNEY 2001}

V. Gaffney, S. Čače, B. Kirigin, P. Leach, N. Vujnović, K. Wardle, D. Wardle: Enclosure and Defence: the Context of Mycenaean Contact with Central Dalmatia, in: V. Karagheorghis, C.E. Morris (Hrsg.): Defensive Settlements of the Aegean and the Eastern Meditteranean after c. 1200 B.C. (Nicosia 2001) 137-156.

\section{GAFFNEY 2002}

V. Gaffney, S. Čače, J. Hayes, B. Kirigin, P. Leach, N. Vujnović: Secret Histories: The Pre-Colonial Archaeological Context for Greek Settlement of the Central Adriatic Islands, in: N. Cambi, S. Čače, B. Kirigin (Hrsg.): Greek influence along the East Adriatic Coast (Split 2002) 25-43.

\section{GARAŠANIN 1966}

M. Garašanin, Moenia aeacia, in: Starinar 17, 1966, 27-28.

\section{GARAŠANIN 1975}

M. Garašanin, Agglomérations fortifiées dans la région frontière de l'est du territoire illyrien, in: A. Benac (Hrsg.), Utvrđena ilirska naselja. Međunarodni kolokvij, Mostar 24-26 oktobar 1974 (Agglomérations fortifiées illyriennes. Colloque international, Mostar 24-26 octobre 1974) 113-120.

\section{GOVEDARICA 2006}

B. Govedarica, Finds of the Cetina-type in the Western Balkan hinterland and the issue of culture-historical interpretation in the prehistoric archaeology, in: VAHD 99, 2006, 27-41.

\section{HÄNSEL 2005}

B. Hänsel, Das alte Istrien, in: Ž. Ujčić (Hrsg.), Histria Istra Istrien. Ein archäologisches Juwel in der Adria (Zagreb 2005) 11-18.

\section{ILAKOVAC 1962}

B. Ilakovac, Prilog arhitekturi i urbanizmu Iadera, in: Rad Ak Zadar 9, 1962, 219-249.

\section{ILAKOVAC 1998}

\section{KOLB 2005}

B. Ilakovac, Liburnska i Rimska Aenona (Nin), in: Radovi 24, 1998, 1-14.

\section{KOLEGA 2001}

F. Kolb, Die Stadt im Altertum (München 2005).

M. Kolega, Nin. Arheološka istraživanja ispred župne crkve sv. Asela

\section{KOLEGA 2005} (Anselma), in: Obavijesti 33, 2001, Nr. 2, 83-95.

M. Kolega, Nin: Nastavak sustavnih iskopavanja na lokalitetu Banovac, in: Obavijesti 37, 2005, Nr. 1, 90-97.

\section{KORKUTI 1975}

M. Korkuti, Alcune charatteristiche degli abitati protourbani nella Iliria meridionale, in: A. Benac (Hrsg.), Utvrđena ilirska naselja. Međunarodni kolokvij, Mostar 24-26 oktobar 1974 (Agglomérations fortifiées illyriennes. Colloque international, Mostar 24-26 octobre 1974) 131-136.

\section{KORKUTI 1988}

M. Korkuti, Illyrien in der Vorgeschichte, in: A. Eggebrecht (Hrsg.), Albanien. Schätze aus dem Land der Skipetaren (Mainz 1988) 7-32. 


\section{KOROŠEC 1980}

J. Korošec - P. Korošec, Istraživanje na Bribirskoj glavici u Bribiru - Les fouilles dans le site de Bribirska glavica a Bribir, in: Diadora 9, 1980, 95-164.

\section{KUNTIĆ MAKVIĆ 2007}

B. Kuntić Makvić, Die Illyrer. Zwischen Griechenland und Italien, in: M.

Sanader (Hrsg.), Kroatien in der Antike (Mainz 2007) 20-23.

\section{LETZNER 2005}

W. Letzner, Das römische Pula, Bilder einer Stadt in Istrien (Mainz 2005) 89101.

\section{LIEBL - WILBERG 1908}

H. Liebl - W. Wilberg, Ausgrabungen in Asseria, in: JÖAI 11, 1908, Beibl. 18-88.

\section{MARCHESETTI 1983}

C. Marchesetti, I castellieri preistorici di Trieste e della regione Giulia (Trieste 1903, reprint 1983).

MARIĆ 1974

Z. Marić, Prahistorijska i protohistorijska utvrđenja na području Daorsa, in: A. Benac (Hrsg.), Utvrđena ilirska naselja. Međunarodni kolokvij, Mostar 24-26 oktobar 1974 - Agglomérations fortifiées illyriennes. Colloque international, MARIĆ 1995 Mostar 24-26 octobre 1974) 103-112.

Z. Maric, Die hellenistische Stadt oberhalb Ošanići bei Stolac (Ostherzegowina), in: BRGK 76, 1995, 31-72.

\section{MARIN 1968/69}

E. Marin, Neke značajke rimske sakralne arhitekture na istočnom Jadranu (Certaine caracteristique de L'architecture religieuse romaine sur le littoral oriental de l'Adriatique, in: VAHD 70/71, 1968/69, 155-176.

\section{MAROVIĆ - ČOVIĆ 1983}

I. Marović - B. Čović, Cetinska kultura, in: S. Gabrovec (Hrsg.), Praistorija Jugoslavenskih Zemalja 4, Bronzano doba (Sarajevo 1983) 191-231.

\section{MATIJAŠIĆ 1990}

R. Matijašić, Breve nota sui templi forensi di Nesazio e Pola, in: La città nell' Italia settentrionale in età romana (Roma 1990) 635-652.

\section{MATIJAŠIĆ 1998}

R. Matijašić, Nesactium (Pula 1998).

\section{MIHOVILIĆ 2005}

K. Mihovilić Baumeister und Herrschaftseliten. Bronzezeit, in: Ž. Ujčić (Hrsg.), Histria Istra Istrien. Ein archäologisches Juwel in der Adria (Zagreb 2005) 31-36.

\section{MIJOVIĆ - KOVAČEVIĆ 1975}

P. Mijović - M. Kovačević, Gradovi i Utvrđenja u Crnoj Gori (Beograd-Ulcinj 1975).

\section{OLUJIĆ 2007}

B. Olujić, Povijest Japoda (Zagreb 2007).

\section{PARZINGER 1991}

H. Parzinger, Archäologisches zur Frage der Illyrer, in: BRGK 72, 1991, 205261.

\section{SANADER 2007}

M. Sanader (Hrsg.), Kroatien in der Antike (Mainz 2007). 
SANADER 2009

M. Sanader, Dalmatia. Eine römische Provinz an der Adria (Mainz 2009).

STARAC 1999

A. Starac, Rimsko vladanje u Histriji i Liburniji I (Pula 1999).

STARAC 2000

SUIĆ 1968

A. Starac, Rimsko vladanje u Histriji i Liburniji II (Pula 2000).

SUIĆ 1975

M. Suić, Bribir (Varvaria) u antici, in: Starohrv. Prosvj. III, 10, 1968, 217-234.

M. Suić, Approche scientifique et méthode de recherche des habitat autochtones sur le territoire illyrien, in: A. Benac (Hrsg.), Utvrđena ilirska naselja. Međunarodni kolokvij, Mostar 24-26 oktobar 1974 - Agglomérations

SUIĆ 2003 fortifiées illyriennes. Colloque international, Mostar 24-26 octobre 1974) 9-36.

M. Suić, Antički grad na istočnom Jadranu (Zagreb 2003, 2. Auflage).

\section{ŠAŠEL KOS 2005}

\section{ŠKEGRO 2000}

M. Šašel Kos, Appian and Illyricum (Ljubljana 2005).

A. Škegro, Bergbau der römischen Provinz Dalmatiens, Godišnjak Centra za balkanološka ispitivanja Akademije nauka i umjetnosti Bosne i Hercegovine XXXI / 29, 2000, 53-176.

TRUHELKA 1893

\section{UJČIĆ 2005}

Ć. Truhelka, Hügelgräber und Ringwälle auf der Hochebene von Glasinac, in: WMBH 1, 1893, 61-112.

Ž. Ujčić (Hrsg.), Histria Istra Istrien. Ein archäologisches Juwel in der Adria (Zagreb 2005).

\section{WILKES 1969}

\section{WILKES 1992}

J. Wilkes, Dalmatia (London 1969).

J. Wilkes, The Illyrians (Oxford 1991).

\section{WILKES - FISCHER-HANSEN 2004}

J. Wilkes - Th. Fischer-Hansen, The Adriatic, in: M.H. Hansen - T.H. Nielsen, An inventory of archaic and classical poleis (Oxford 2004) 321-22.

\section{ZANINOVIĆ 2005}

M. Zaninović, Zemljopisno-povijesni položaj luka Parentija i Nezakcija Geographical and historical positions of the ports of Parentium and Nesactium, in: Histria archaeologica 36, 2005, 115-136.

\section{ZANINOVIĆ 2007}

M. Zaninović, Die römische Zeit, in: M. Sanader (Hrsg.), Kroatien in der Antike (Mainz 2007) 61-71. 
Frankfurter elektronische Rundschau zur Altertumskunde 10 (2009)

\section{Abbildungsnachweise}

Abb. 1. SANADER 2007, 25, Abb. 19.

Abb. 2. MARIĆ 1974, T. 1, 2.

Abb. 3. WILKES 1991, 191, Fig. 20.

Abb. 4. ŠAŠEL KOS 2005, 227, Fig. 50.

Abb. 5. BATOVIĆ 1970, S1. 1.

Abb. 6. BENAC 1987, 508.

Abb. 7. CEKA 1988, Abb. 8.

Abb. 8. LIEBL - WILBERG 1908, 19, Abb. 2.

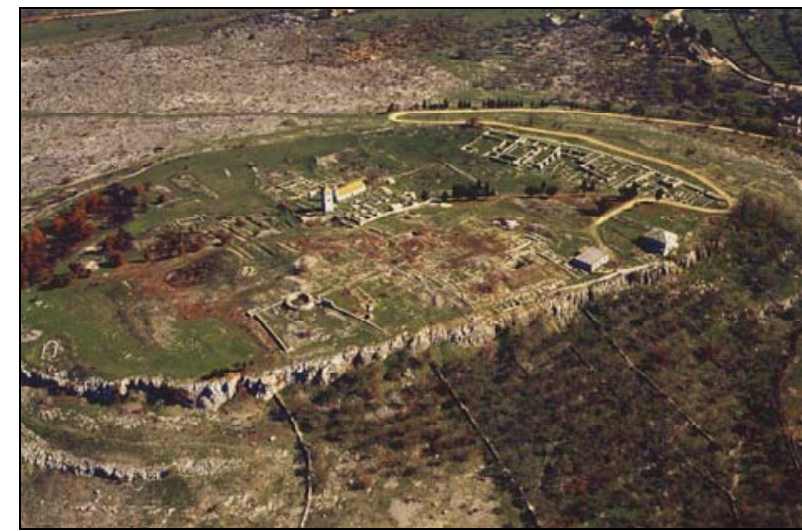

Abb. 1. Beispiel Gradina-Anlage, Varvaria (Bribir, Kroatien), bronze- und eisenzeitliche liburnische Siedlung, später Municipium Varvariae

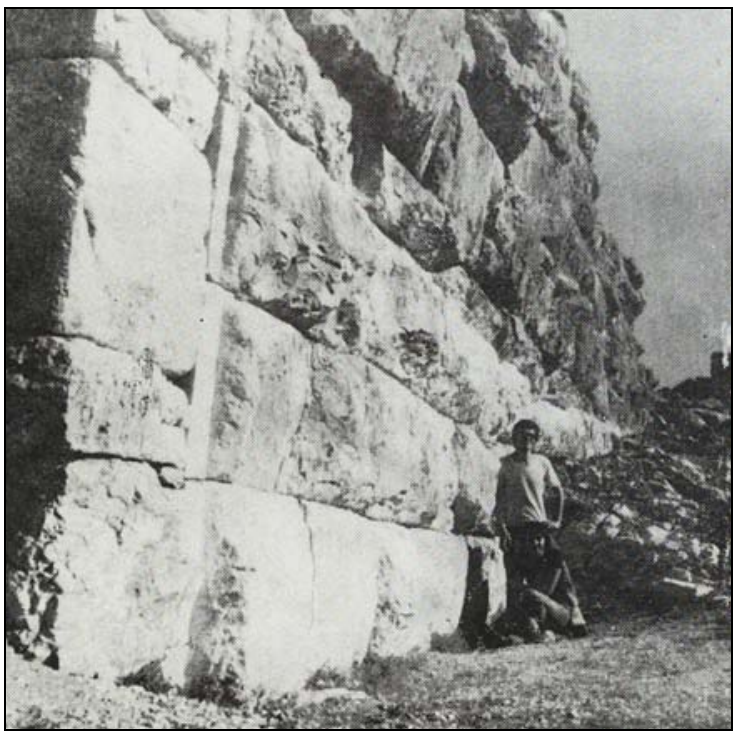

Abb. 2. Beispiel Gradina-Mauern, "Daorson" (Ošanići, Bosnien und Herzegowina) 
Frankfurter elektronische Rundschau zur Altertumskunde 10 (2009)
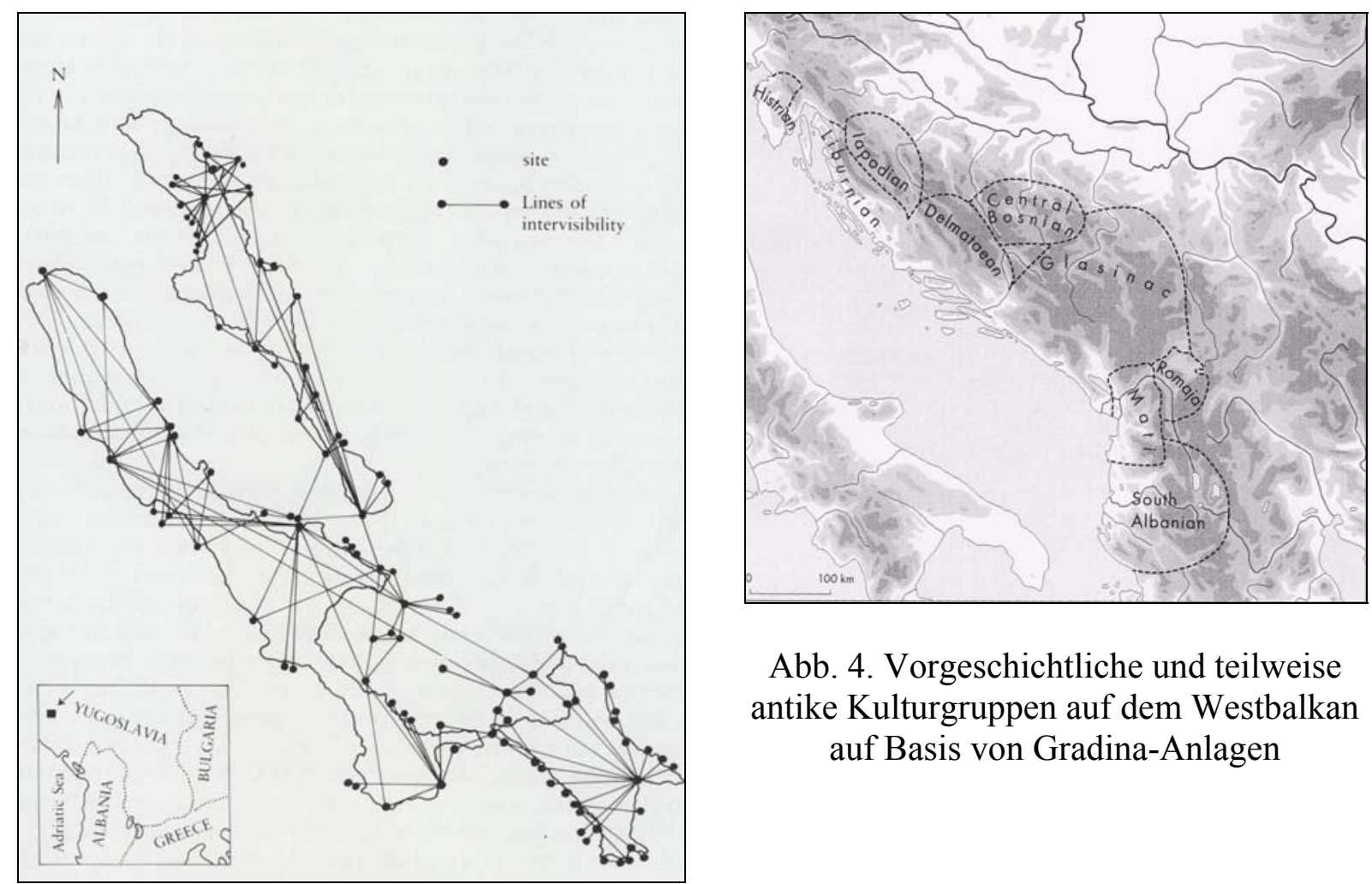

Abb. 4. Vorgeschichtliche und teilweise antike Kulturgruppen auf dem Westbalkan auf Basis von Gradina-Anlagen

Abb. 3. Gradina-Anlagen in den Hochebenen von Glamoč, Livno und Duvno (Bosnien und Herzegowina), Raumkonzeption und Sichtachsen

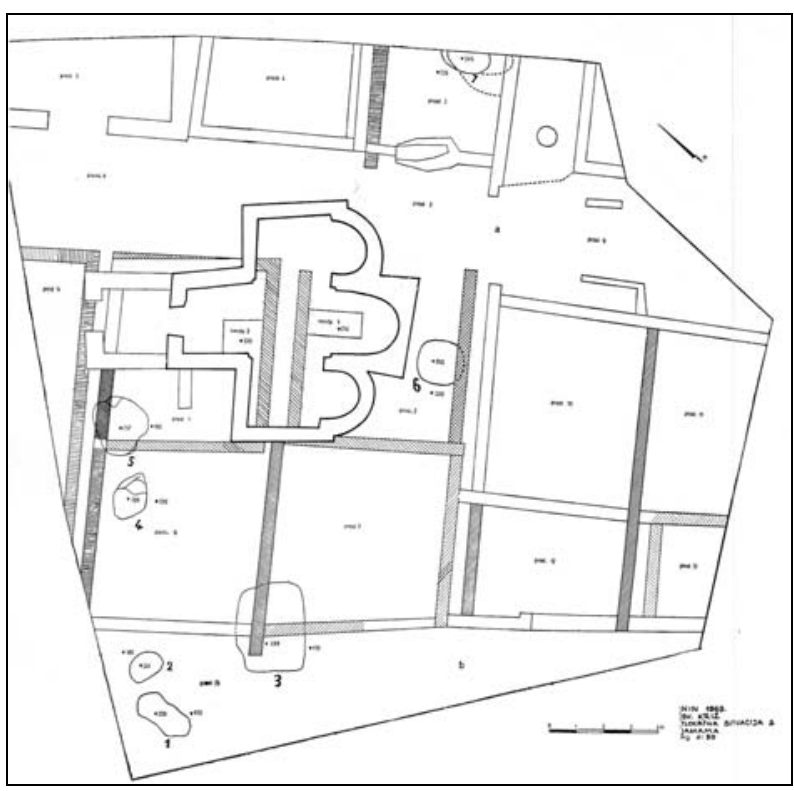

Abb. 5. Aenona (Nin, Kroatien), liburnische (gestrichelt) und römerzeitliche Hausfundamente (weiß) entlang zwei Straßenzügen (Detail)

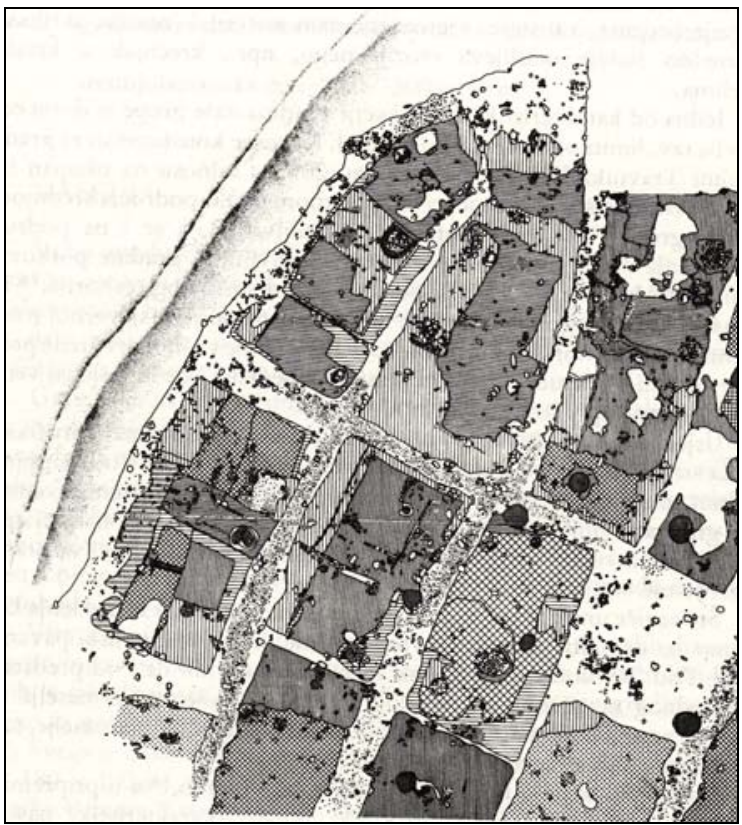

Abb. 6. Gradina bei Pod (Bosnien und Herzegowina), Infrastruktur, Detail 
Frankfurter elektronische Rundschau zur Altertumskunde 10 (2009)

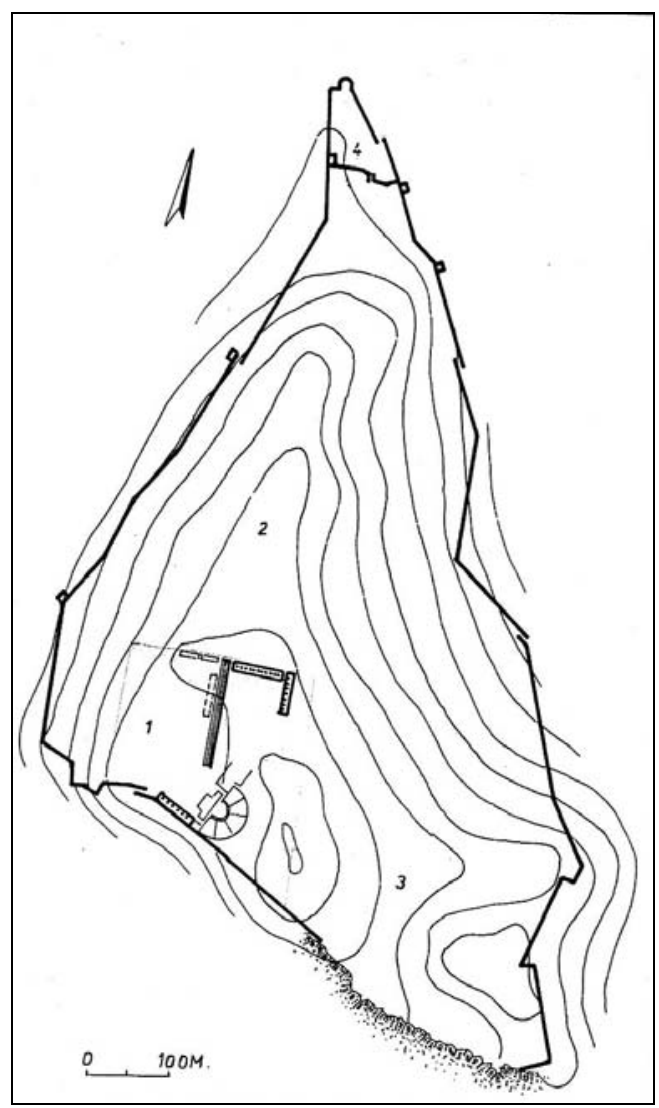

Abb. 7. Byllis (Albanien), Stadtanlage

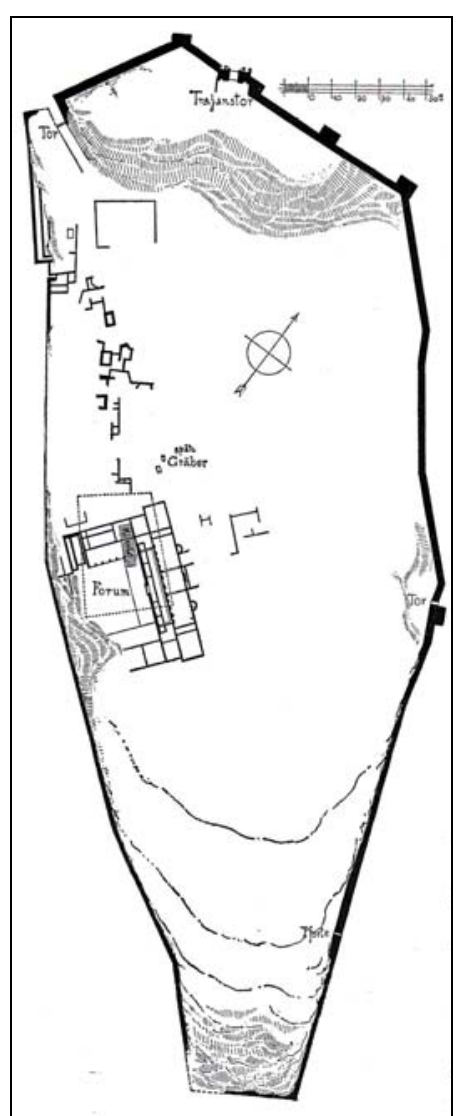

Abb. 8. Asseria (Kroatien), Stadtanlage 